\title{
Numerical Simulation and Experimental Validation of Calibrant- Loaded Extraction Phase Standardization Approach
}

\author{
Md. Nazmul Alam and Janusz Pawliszyn* \\ Department of Chemistry, University of Waterloo, Waterloo, Ontario N2L 3G1, Canada \\ Supporting Information
}

ABSTRACT: We present the kinetics of calibrant release and analyte uptake between the sample and calibrant-loaded extraction phase, CL-EP, with a finite-element analysis (FEA) using COMSOL Multiphysics software package. Effect of finite and infinite sample volume conditions, as well as various sample environment parameters such as fluid flow velocity, temperature, and presence of a binding matrix component were investigated in detail with the model in relation to the performance of the calibration. The simulation results supported by experimental data demonstrate the suitability of the CL-EP method for analysis of samples with variation of the sample environment parameters. The calibrant-loaded approach can provide both total and free concentrations from a single experiment based on whether the partition coefficient $\left(K_{\text {es }}\right)$ value being used is measured in a matrix-matched sample or in a matrix-free sample, respectively. Total

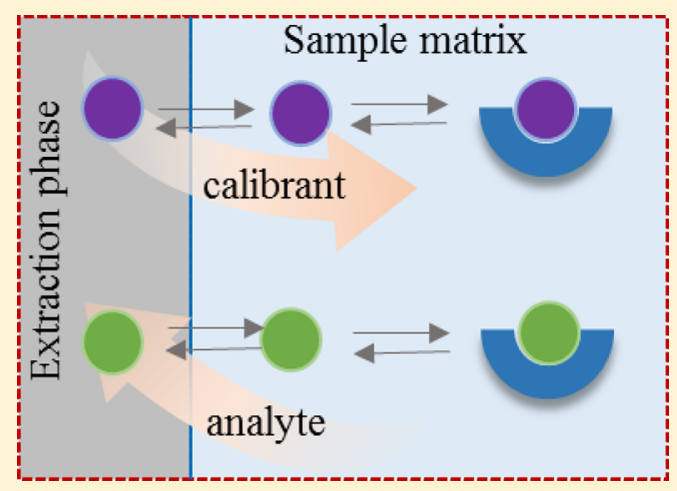
concentrations can also be obtained by utilizing CL-EP in combination with external matrix-matched calibrations, which can be employed to automate the sampling process and provide corrections for variations in sample preparation, matrix effects, and detection processes. This approach is also suitable for very small volumes of sample, where addition of an internal standard in the sample is either troublesome or can change the sample characteristics.

The he fundamental of the solid-phase microextraction (SPME) technique is based on exposing a small amount of extraction phase (extractant) to a sample for a predetermined length of time. The extraction of analyte mainly governed by the preferential affinity of the analytes to the extractant. The higher the affinity the analyte has for the extractant relative to the sample matrix, the greater the uptake amount of analyte. If the extractant is exposed for long enough to attain a concentration equilibrium between the extractant and sample, the net uptake amount remains unchanged after the equilibration is reached. Therefore, in SPME, the goal is not to extract $100 \%$ of the analyte from a sample unlike other conventional sample preparation techniques.

In SPME, the mathematical correlation, also called "calibration", between the extracted amount of target analytes on an extraction phase and their concentration in the sample matrix is quite straightforward when the two-phase system reaches equilibrium. Typically, determinations of analyte concentrations at equilibrium conditions are conducted under certain conditions: with the use of very thin extractant with low extraction capacity, for analytes with low to moderate partition coefficients or with very high sample agitation conditions. ${ }^{2}$ However, when sampling certain analytes in slow agitation samples, such as sampling of polycyclic aromatic hydrocarbons (PAHs) from aquatic environments, unfeasibly long periods may be needed to transport enough analytes to the extractant through the aqueous boundary layer and, thus, reach equilibrium. In addition, aiming to reach equilibrium under such long exposure times in real sample matrixes can result in deterioration of the extractant, owing to unwanted interactions with sample matrix components in vivo or in situ. In order to avoid long equilibration times as well as increase measurement accuracy, an alternative pre-equilibrium calibration approach has been proposed by Chen et $\mathrm{al}^{3}$ The pre-equilibrium calibration method is based on the concurrent desorption of a chemical species previously loaded onto the extraction phase while extraction occurs under the same experimental conditions. The preloaded species should have similar physicochemical properties to the target analyte and must not be present in the sample matrix. In this method, the loaded chemical is assumed to follow a desorption kinetics model that is identical to the extraction kinetics of the target analyte from the sample matrix. In the kinetic regime of the extraction profile, this method of calibration has been called "kinetic calibration", 3 "on-fiber standardization", 4 or stable isotope solid-phase microextraction (SI-SPME). ${ }^{5}$ Although the method was first utilized with poly(dimethylsiloxane) PDMS-based SPME, a number of different sorbents with different geometries have been studied, such as porous particle-based SPME, liquidphase microextraction (LPME), hollow fiber-protected liquidphase microextraction (HF-LPME), stir bar microextraction (SBME), etc. ${ }^{6}$ For simplicity, this calibration technique is named as calibrant-loaded extraction phase (CL-EP). This

Received: May 8, 2016

Accepted: August 10, 2016

Published: August 10, 2016 
kinetic calibration method has been extended to many applications, showing that the method compensates for variations in experimental conditions. For example, Zhan et al. ${ }^{7}$ demonstrated that the calibrant-loaded SPME approach can compensate for the effect of matrix tortuosity and protein binding. However, during pre-equilibrium extraction, small variations in experimental conditions such as sample volume, temperature, agitation, binding matrix components, and sampling time have been noted to sometimes result in significant experimental error. ${ }^{8}$

It is difficult to experimentally test the suitability of different calibrants for a wide range of analyte properties. Recently, we developed a computational model for the mass transport processes in SPME. ${ }^{9}$ The model considers the extraction phase as having an analyte concentration that is equal to zero in the beginning of the extraction process. Here, diffusion only transport in the extraction phase is assumed, while analyte transport in the sample matrix is assumed to occur by convection and diffusion coupling with a reversible reaction to a binding matrix component present in sample. The model results suggest that the extraction kinetics are dependent on a number of parameters, including the concentration of the binding matrix and the binding affinity of the analyte to the matrix. These findings served as the primary motivation for the present study, which focuses on the study of the desorption kinetics of a calibrant that is preloaded on the extraction phase prior to deployment to the sample matrix. While the study of chemical release from preloaded materials to different phases remains an active field of research, such as in areas that focus on research related to drug delivery ${ }^{10}$ and performance reference material $(P R C)^{11}$ based calibration, modeling of the quantitative relationship between the release and sorption is limited.

The aim of this study was to develop a mathematical model and computational simulation to estimate calibrant desorption and analyte sorption kinetics, the data for which can be used to facilitate the selection of calibrants for a variety of applications. Moreover, the effects of various environmental conditions such as hydrodynamics, temperature, and the presence of a binding matrix have been characterized for CL-SPME quantification.

\section{EXPERIMENTAL SECTION}

Mathematical Models of Calibrant-Loaded SPME (CLSPME). We have recently described a computational model that accounts for processes occurring during extraction by an SPME coating, namely the transport and reaction within the sample matrix. ${ }^{9}$ In the present work, we use the same model for extraction. However, for desorption of calibrant, the calibrant is assumed to be present in the extraction phase, where transport occurs only by diffusion. Considering that the model has been described in detail in our previous work, ${ }^{9}$ it will be only briefly overviewed here, highlighting its most important features. As depicted in Figure S1, the model considered a two-dimensional segment of a sample-extractant system. The flow in the sample domain is governed by the Navier-Stokes equation, while the flow field is treated as steady. Time-dependent analyte or calibrant transport occurs as follows: (1) the loaded calibrant diffuses through the coating layer and, due to a concentration jump, a mass flux is established across the interface, where the calibrant being transferred to the adjacent sample medium; (2) in the sample matrix, chemicals transport via convection and diffusion, with specific binding and unbinding to the binding matrix component taking place. For the interaction of calibrant or analyte with the binding matrix, a nonlinear saturable reversible binding model is considered. The reaction shown in eq $1^{12}$ describes a $1: 1$ reversible and saturable binding for a matrix component $(\mathrm{M})$ with analyte or calibrant (A).

$$
\mathrm{A}+\mathrm{M} \underset{k_{\mathrm{d}}}{\stackrel{k_{\mathrm{a}}}{\rightleftarrows}} \mathrm{AM}
$$

In this reaction, $k_{\mathrm{a}}$ and $k_{\mathrm{d}}$ represent the second-order association (binding) rate constant and first-order dissociation (unbinding) rate constant, respectively, for the interaction of $\mathrm{M}$ with A. The ratio $\left(k_{\mathrm{a}} / k_{\mathrm{d}}\right)$ is defined as the association equilibrium constant $\left(K_{\mathrm{a}}\right)$ for this system. The mathematical equations for the binding of calibrant and analyte to the matrix components are the same as reported previously. ${ }^{9}$

\section{NUMERICAL METHODS}

COMSOL Multiphysics 5.1, a finite element method (FEM) based software package, was utilized in this study to analyze the mass transfer processes in CL-SPME. In order to obtain an accurate representation of the SPME system, the timedependent partial differential equations for each of these physical processes must be solved simultaneously. The procedure used to solve this problem is divided into two steps: (1) determination of the fluid velocity profile at steadystate, assuming incompressible flow, and (2) use of this steadystate velocity profile as the initial condition to solve for the coupled transient mass transport and sorption equations. The rate constants $a_{\mathrm{e}}$ and $a_{\mathrm{d}}$ can be obtained through eq $2^{13}$ and eq $3,{ }^{3}$ respectively, if the initial amount $\left(q_{0}\right)$ of calibrant loaded onto the coating, sampling time $t$, and the quantity extracted at equilibrium are known.

$$
\frac{n}{n_{\mathrm{e}}}=1-\exp \left(-a_{\mathrm{e}} t\right)
$$

where $t$ is the exposure time of the coating within the sample, $n$ is the extracted amount (usually in $\mathrm{ng}$ ) at time $t, n_{\mathrm{e}}$ is the extracted amount (nanogram) at equilibrium, and $a_{\mathrm{e}}$ is the extraction rate constant that describes how fast equilibrium can be attained and is determined by the mass-transfer coefficients, the distribution constant, as well as the physical dimensions of the sample matrix and the SPME sampler

$$
\frac{Q}{q_{0}}=\exp \left(-a_{\mathrm{d}} t\right)
$$

where $Q$ is the amount of calibrant remaining on the coating after retraction of the coating from the sample matrix.

\section{RESULTS AND DISCUSSION}

Desorption Kinetics of Loaded Calibrant. Soon after the extraction phase comes into contact with the sample matrix, analytes are transported from the sample matrix and into the extraction phase, while the calibrant preloaded on the extraction phase releases into the sample. ${ }^{3}$ Fractions of calibrants with the fixed diffusion coefficient but different partition coefficients $\left(K_{\mathrm{es}}\right)$ released from the preloaded coating are shown in Figure 1. The release of chemicals with high $K_{\mathrm{es}}$ proceeds more slowly than that of chemicals with a low $K_{\mathrm{es}}$. For the finite volume sample shown in Figure 1a, complete release did not occur for even the lowest $K_{\mathrm{es}}$ calibrant. As the calibrant is released into the finite volume sample, the calibrant concentration builds up in the external volume, allowing for local equilibrium to be established between the extraction phase 
(a)

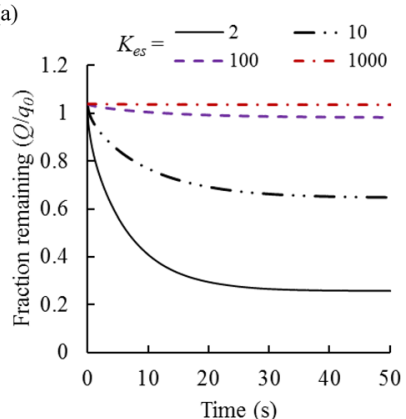

(b)

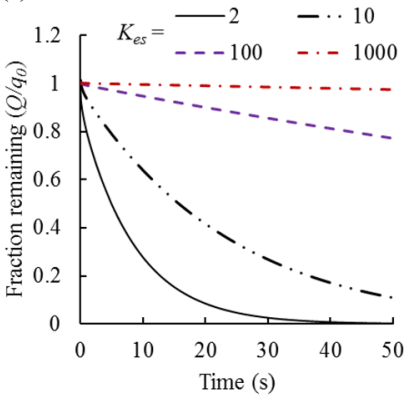

Figure 1. Fractions of calibrants remaining on the extraction phase at different partition coefficients $\left(K_{\mathrm{es}}\right)$ (a) finite sample volume, flow velocity $=0 \mathrm{~cm} \mathrm{~s}^{-1}$ and (b) infinite sample volume with flow velocity of $0.1 \mathrm{~cm} \mathrm{~s}^{-1}$. For both the cases, the absence of a binding matrix component is assumed. Coating thickness was $45 \mu \mathrm{m} ; D_{\mathrm{s}}\left(7.33 \times 10^{-6}\right.$ $\mathrm{cm}^{2} \mathrm{~s}^{-1}$ ) was considered for all the calibrants so that $\delta_{\mathrm{s}}$ does not vary by the compound, $D_{\mathrm{e}}=D_{\mathrm{s}} / 6$.

and the sample. ${ }^{14}$ As an apparent equilibrium is established, further release of calibrant from the extraction phase comes to a halt. In addition, lower fractions are expected to be desorbed with calibrants of higher $K_{\mathrm{es}}$. On the other hand, for infinite sample volumes, the release proceeds either to completeness (for low $K_{\mathrm{es}}$ ) or linearly decreases (for high $K_{\mathrm{es}}$ ) to reach full desorption from the extraction phase (Figure $1 \mathrm{~b}$ ). This is owing to the fact that the concentration of calibrant in an infinite sample medium never increases due to the existence of perfect sink conditions in the sample domain at any time of sampling. Since the SPME-sample system is primarily controlled by the diffusion boundary layer $\left(\delta_{s}\right)$, the magnitude of the $K_{\mathrm{es}}$ is the most important driving force of desorption kinetics. Although $\delta_{\mathrm{s}}$ is assumed to vary with compound diffusivity by a factor of $\left(D_{s}\right)^{1 / 3},{ }^{15}$ aqueous diffusivity usually does not vary in most cases. ${ }^{16}$ Even in cases where a difference in diffusivity between compounds is present, the primary driver of release kinetics is the difference in partition coefficients (for effect of diffusion coefficients, see Figure S2). More discussion on the effect of diffusivities is included in a later section of this work.

The simulation results suggest that the choice of calibrant for a given application should be primarily made based on the partition coefficient of the calibrant. If too much calibrant is released too quickly, it may have a toxic (for in vivo sampling) or short-term effect on the sample matrix. On the other hand, if the calibrant is released too slowly, then the remaining quantity may not differentiate with the initial load. From a practical point of view, it is often impossible to evaluate the full sorption/desorption time profiles of chemicals with a high log $K_{e s}(>5)$, owing to the extremely long equilibrium times of such target analytes and the very low desorption rates for calibrants in the coating. ${ }^{17}$ If the release of calibrant from the coating is too slow to allow for a statistical evaluation of the extraction or desorption kinetics, the estimated rate constant values $\left(a_{\mathrm{d}}\right)$ will be poor and statistically not significantly different from zero. CL-SPME is a practicable extraction technique only for compounds for which significant desorption can be measured within the experimental time period. In contrast, the mechanistic model can be employed to obtain the fraction of analytes accumulated or dissipated at any point of the sampler deployment period for any pair of analyte and calibrant. The currently proposed model allows for the prediction of a reasonable offload amount suitable for SPME calibration.
Iso-Symmetry between Extraction and Desorption and Model Validation. Although the CL-SPME approach has been employed for the equilibrium regime of extraction, ${ }^{18}$ most applications were in the kinetic regime owing to the short sampling times afforded by the technique. The main assumption of the kinetic calibration approach is that the desorption of calibrant must follow kinetics similar to the uptake kinetics of the corresponding analytes. In order to show iso-symmetry, the extracted amount and the calibrant remaining on the extraction phase are normalized by the amount at equilibrium and the loaded amount, respectively, as plotted in Figure 2. In Figure 2a, previously published
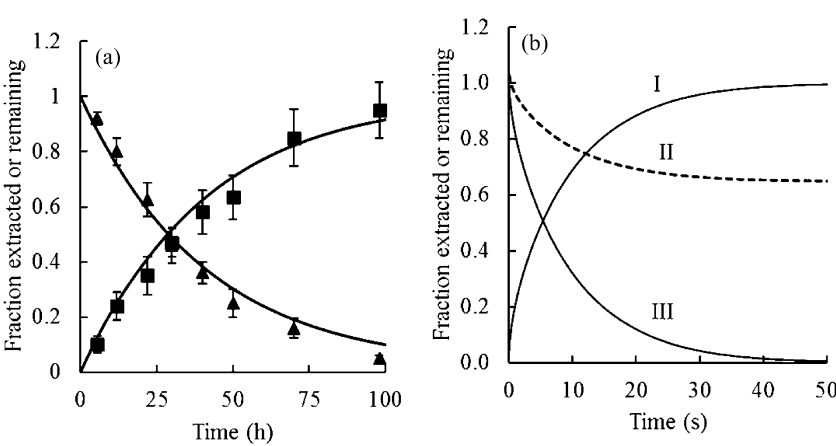

Figure 2. Iso-symmetry of sorption and desorption in calibrant-loaded SPME. (a) Simultaneous sorption of pyrene ( $\square$ ) onto the PDMS coating from the flow-through system and desorption of deuterated pyrene $(\boldsymbol{\Delta})$ from the PDMS coating into the flow-through system. The data points are the experimental values and the lines are obtained from the numerical simulations by employing the parameters corresponding to the chemicals used in the experiments; $K_{\mathrm{es}}{ }^{\mathrm{A}}$ and $K_{\mathrm{es}}{ }^{\mathrm{C}}=40738, D_{\mathrm{s}}{ }^{\mathrm{A}}, D_{\mathrm{s}}{ }^{\mathrm{C}}=6.59 \times 10^{-6}$ and flow velocity $=3 \mathrm{~cm} / \mathrm{s}$. (b) The iso-symmetric behavior for a finite volume sample that needs correction to account for local equilibrium: (I) extraction profile of an analyte, (II) desorption profile of the calibrant, and (III) desorption profile of the calibrant after correction with eq 5 . Parameters are same as shown in Figure 1.

experimental data was replotted along with the model simulation results for a d8-pyrene loaded PDMS fiber exposed to a flowing pyrene aqueous solution for different extraction times. ${ }^{19}$ Very good fitting of the experimental data validates the numerical model used in this work; see Table S6 for a quantitative evaluation of the agreement between the model and the experiment.

The iso-symmetric behavior of sorption and desorption can be recognized by the intersection point of the two time profiles at around 0.5 of the $y$ axis. In other words, $50 \%$ extraction and desorption are achieved at the same time of deployment in the sample matrix (in this example, the elapsed time is approximately $30 \mathrm{~h}$ ). With the availability of iso-symmetric sorption and desorption time profiles for a pair of analyte and calibrant, one can easily calculate the concentration of analyte in a sample matrix at practically any point of the time profile with the use of eq $4:^{3}$

$$
C_{\mathrm{s}}=\frac{n}{K_{\mathrm{es}} V_{\mathrm{e}}\left(1-\frac{Q}{q_{0}}\right)}
$$

where $C_{\mathrm{s}}$ is the analyte concentration in the sample and $V_{\mathrm{e}}$ is the volume of the extractant. On the other hand, iso-symmetric behavior may not exist for finite volume samples as shown in 
Figure $2 \mathrm{~b}$. Therefore, the iso-symmetic profiles for sample with small volume can be obtained by using the following equation:

$$
\frac{\mathrm{Q}-q_{\mathrm{e}}}{q_{0}-q_{\mathrm{e}}}=\exp \left(-a_{\mathrm{d}} t\right)
$$

where $q_{\mathrm{e}}$ is the quantity of calibrant remaining on the extraction phase after local equilibrium is reached. Next, the computational model was employed to study the effect of a few parameters that might affect the desorption kinetics and, consequently, the iso-symmetry of desorption and sorption.

Effect of $K_{\mathrm{es}}$ on Desorption Rate Constant $\left(a_{\mathrm{d}}\right)$. The influence of the wide range of partition coefficient $\left(K_{\mathrm{es}}\right)$ values on the desorption rate constant is predicted with the proposed model. Figure 3 depicts how the $a_{\mathrm{d}}$ significantly decreases with

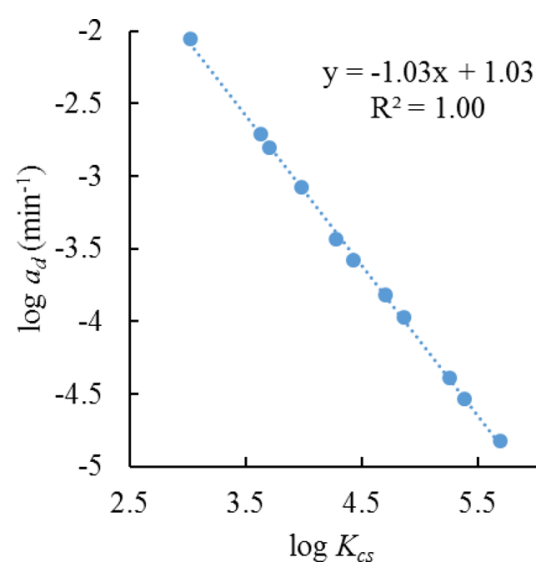

Figure 3. Desorption rate constant, $a_{\mathrm{d}}$ obtained by varying the coating-sample partition coefficient $\left(K_{\mathrm{es}}\right)$ by keeping all other conditions the same as Figure 1.

the increase of $K_{\mathrm{es}}$. For this simulation, sampling time was chosen to be equivalent to the time needed for up to $50 \%$ of the calibrant to desorb from the extractant, since equilibration times vary widely for the wide range of $K_{\mathrm{es}}$ values of the PAHs used in this study. The numerical simulation estimated an approximate 3-fold decrease of $a_{\mathrm{d}}$ relative to a 3-fold increase in $K_{\text {es }}$. A similar change in the coefficients was obtained by plotting the experimentally obtained $a_{\mathrm{d}}$ versus $K_{\mathrm{es}}$ reported by two different groups (see Figure S3). It should be emphasized here that this trend might surprise some scientists who are familiar with other passive sampling devices where the mass transfer coefficient $\left(k_{\mathrm{m}}\right)$ is usually plotted against the partition coefficients. For such systems, Huckins et al. ${ }^{20}$ proposed that the mass transfer coefficient and the diffusion coefficient are of the form $k_{\mathrm{m}} \sim D^{2 / 3}$. On the basis of this relationship, only a $30 \%$ variation in the mass transfer coefficients of the calibrants was obtained. In contrast, the sharp decreasing trend of $a_{\mathrm{d}}$ with varied $K_{\mathrm{es}}$ cannot be explained only by considering the variation of diffusivities among the calibrants. Additionally, the $a_{\mathrm{d}}$ used in the CL-SPME relates to the mass transfer coefficient, $k_{\mathrm{m}}$, according to the following equation: ${ }^{21}$

$$
a_{\mathrm{d}}=\frac{A k_{\mathrm{m}}}{K_{\mathrm{es}} V_{\mathrm{e}}}
$$

where $A$ and $V_{\mathrm{e}}$ are the area and volume of the extractant, respectively. Therefore, for a given calibrant, the $a_{\mathrm{d}}$ is a function of not only the $k_{\mathrm{m}}$ but also the $K_{\mathrm{es}}$. This implies that the steep decrease of $a_{\mathrm{d}}$ is due to the inclusion of the $k_{\mathrm{m}}$ and the partition coefficients $\left(K_{\mathrm{es}}\right)$ in the calculations. The simulation results support the fact that the initial mass transfer rate is influenced by both the diffusivity and $K_{\mathrm{es}}$ of respective calibrants.

Effect of Flow Velocity. Agitation of the sample matrix decreases the boundary layer thickness, which should enhance the mass transfer kinetics for both the sorption and desorption processes. This phenomena was first investigated by exposing the calibrant-loaded extraction phase to a sample matrix at various hydrodynamic conditions. The model simulations were conducted at flow rates for which the flow was characterized as laminar for the majority of the sample domain. This was checked by observing no vortices behind the SPME coating (see Figure S4). Figure 4a shows the effect of flow velocity on
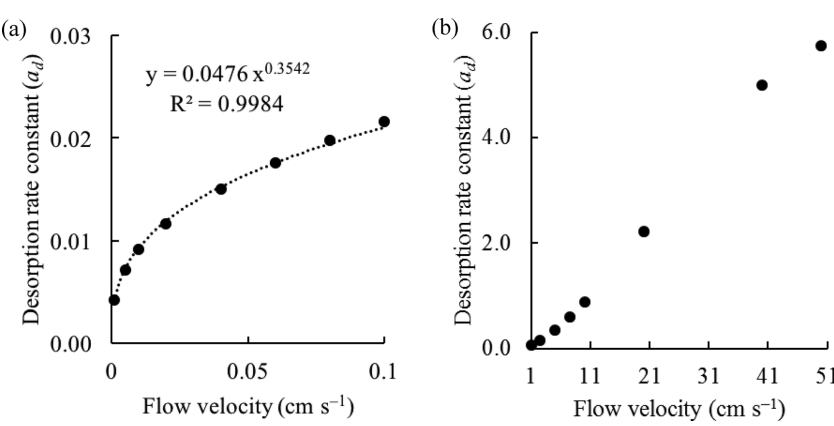

Figure 4. Dependence of $a_{\mathrm{d}}$ as a function of linear sample flow velocity at two flow regimes: (a) laminar flow and (b) turbulence flow. Model simulation was carried out by using $\log K_{\mathrm{es}}=4, D_{\mathrm{s}}=1 \times 10^{-6} \mathrm{~cm} \mathrm{~s}^{-1}$.

the rate constant $a_{\mathrm{d}}$ under laminar flow conditions (0.001-0.1 $\left.\mathrm{cm} \mathrm{s}^{-1}\right)$. A significant increase in $a_{\mathrm{d}}$ was observed until $0.05 \mathrm{~cm}$ $\mathrm{s}^{-1}$, whereas the rate of increase was observed to slow between $0.05 \mathrm{~cm} \mathrm{~s}^{-1}$ and $0.1 \mathrm{~cm} \mathrm{~s}^{-1}$. The exponential fitting of data provided a coefficient of $\sim 0.4$. As shown in Figure $4 \mathrm{~b}$, as the sample flow increases, the $a_{\mathrm{d}}$ increases linearly for all of the tested velocities (up to $50 \mathrm{~cm} \mathrm{~s}^{-1}$ ). Accordingly, an additional increase in $a_{\mathrm{d}}$ with increasing flow rate is observed when the flow regime switches from laminar to turbulent. At a high flow velocity, the Reynolds number is very high, and stable vortices appear behind the SPME coating. As seen in Figure S4b, the vortices significantly affect mass transfer to the coating. Previous reports on other sampling devices also demonstrated similar proportional increases of mass transfer in the case of laminar and turbulent exterior flows, showing that mass transfer is related to velocity to the power of 0.5 and $0.8-0.9$ for laminar and turbulent cases, respectively. ${ }^{22}$ The sample fluid flow velocity affects the desorption and sorption kinetics to the same extent, leaving the calibration unaffected by the change of flow velocity during a sampling period.

Effect of Temperature. The effect of temperature on the transport of chemicals between the extractant and sample matrix is a bit complicated, since both the sample media of the transport and the properties of the chemicals can be affected by temperature. Thus, the change of $a_{\mathrm{d}}$ with temperature was simulated with the computational model and compared with the experimental data obtained from previous published work. ${ }^{3}$ Figure 5 demonstrates that the higher the temperature, the greater the value of $a_{\mathrm{d}}$. With the increase of temperature, the $a_{\mathrm{d}}$ increases owing to the mass-transfer coefficient $\left(k_{\mathrm{m}}\right)$ increase, but the increase is partially offset by the decrease of the distribution coefficient $\left(K_{\mathrm{es}}\right)$ (see eq 6). For this study, the diffusivities and partition coefficients of the analytes were obtained from the literature. ${ }^{3}$ The simulated data provided a 


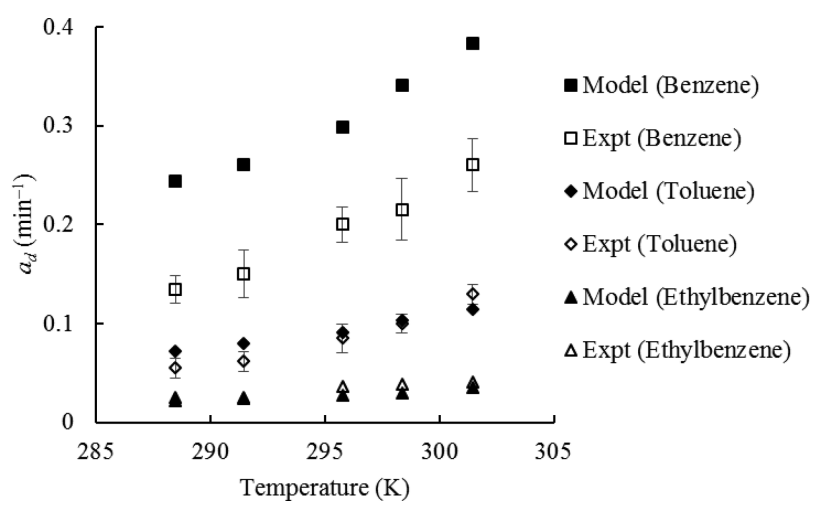

Figure 5. Effect of temperature on the desorption kinetics, $a_{\mathrm{d}}$. Desorption of benzene $(\boldsymbol{\square}, \square)$, toluene $(\diamond, \diamond)$, and ethylbenzene $(\boldsymbol{\Delta}, \triangle)$ from a $100-\mu \mathrm{m}$ PDMS fiber into water at a rate of $0.25 \mathrm{~cm} / \mathrm{s}$ at various temperatures. Model simulation data are shown in filled symbols, whereas the open symbols are used to plot the experimental data.

very good fit with the experimental results; detailed quantitative errors are shown in Table S6. However, the discrepancy between the experimental and model simulation results obtained for benzene is likely due to the $K_{\mathrm{es}}$ value (for benzene, $K_{\mathrm{es}} \approx 60$ ) used in this simulation, as $K_{\mathrm{es}}$ values for benzene have been defined as larger than 100 in other reports. ${ }^{23}$ For extraction, temperature also affects in the same manner and iso-symmetry is preserved. Hence, CL-SPME provides quantitative results even if there is a change in temperature during the sampling period.

Effect of Binding Matrix on the Desorption and Uptake Rate Constants. The kinetics of both the sorption of analytes and desorption of calibrant have been experimentally reported to be affected by the presence of a binding matrix component in a sample. ${ }^{7,9}$ At first, a computational simulation was carried out to study the effect of concentration of a matrix component (for example, albumin) on the desorption kinetics at the finite sample volume (Figure 6a). In the model, the increase in the concentration of the matrix component was shown to enhance the calibrant release kinetics. For instance, $1 \%$ of albumin caused almost all of the calibrant to be released within $30 \mathrm{~s}$, whereas most of the calibrant was shown to remain on the extraction phase if no binding matrix was present in the finite sample volume. This can be explained by the fact that as
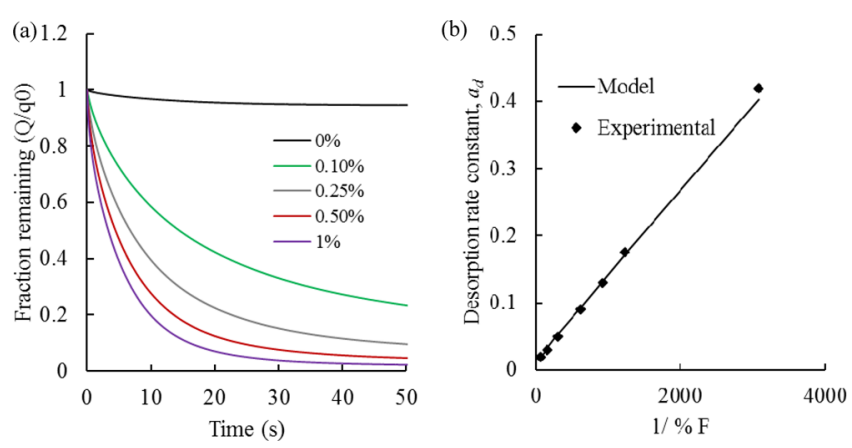

Figure 6. (a) Effect of matrix concentration on desorption kinetics in finite volume case. $K_{\mathrm{es}}=100, K_{\mathrm{a}}=1 \times 10^{5} \mathrm{~L} / \mathrm{kg}, k_{\mathrm{d}}=1 \mathrm{~s}^{-1}$ (labile). (b) The dependence of desorption rate constant, $a_{\mathrm{d}}$, on the free concentration of pyrene present in sample with increasing concentration of a binding matrix (BSA). The free concentration of analyte decreases with the addition of BSA in the sample. binding occurs, a greater concentration gradient is produced in the aqueous boundary layer due to the transfer of free calibrant molecules into their bound form, thus hastening faster calibrant transport from the coating-sample interface. For an infinite sample volume, the $a_{\mathrm{d}}$ was similarly affected by the presence of the binding matrix, although the $a_{\mathrm{d}}$ was much different for matrix-free samples (Figure S5a). This implies that desorption kinetics might be independent of sample volume or agitation for samples containing high concentration of binding matrix components due to the reduced boundary layer thickness. This was further verified by running simulations at two different flow velocities, the results of which are shown in the Supporting Information (see Figure S5b). The obtained results imply that the desorption rate is controlled progressively by the diffusion of calibrant in the extraction phase. Consequently, the extraction-desorption hysteresis observed in the finite volume sample without the presence of a binding matrix was weakened in the matrix-containing sample.

Apart from the concentration of the binding matrix, $a_{\mathrm{d}}$ also depends on the binding affinity of analyte or calibrant with the binding matrix (how tight the binding is at equilibrium). As shown in Figure S6a, the extent of the enhancement observed for the desorption kinetics is lower for $K_{\mathrm{a}}$ values of $1 \times 10^{3}$ liter $/ \mathrm{kg}$ in comparison to the results shown in Figure $6 \mathrm{a}$ for $K_{\mathrm{a}}$ values of $1 \times 10^{5}$ liter $/ \mathrm{kg}$.

The developed computational model was compared with experimental data obtained from Jiang et al. ${ }^{24}$ As shown in Figure $6 \mathrm{~b}$, the experimental results of the enhanced desorption kinetics with increasing concentration of bovine serum albumin (BSA) were accurately predicted with the mathematical model. Although the $a_{\mathrm{d}}$ remains unchanged at very low concentrations of BSA (from $10 \mathrm{ng} / \mathrm{mL}$ to $10^{4} \mathrm{ng} / \mathrm{mL}$ ), the $a_{\mathrm{d}}$ values linearly increase with the decrease in its free concentration, owing to the higher concentrations of the binding matrix (BSA).

The slopes of the dependency of $a_{\mathrm{d}}$ on the concentration of matrix components (similar to Figure $6 \mathrm{~b}$ ) for a number of calibrants with different $K_{\mathrm{es}}$ and $K_{\mathrm{a}}$ values were predicted from the model and compared with reported experimental data, as shown in Table 1 . As can be seen, the computational model predicted very well the variation of $a_{\mathrm{d}}$ for pyrene and phenanthrene; however, large error is seen for acenaphthene and fluoranthene. Since fluoranthene and pyrene have very close $K_{\mathrm{es}}$ and $D_{\mathrm{s}}$ values, the slops should be of close value as well. The discrepancy between the model and experimental data might be due to difficulty in measuring these very high hydrophobic compounds in water sample due to their attachment on the sample vial. For acenaphthene, the large error might be due to the difficulty in determining fraction remaining after very sort sampling time since the equilibration time is less than a minute in the presence of high concentration of BSA used in the experiments.

The $a_{\mathrm{d}}$ is also influenced by the binding kinetics or lability of the calibrant-matrix pair. The dissociation rate constant $\left(k_{\mathrm{d}}\right)$ was varied by keeping the same thermodynamic association constant $\left(K_{\mathrm{a}}\right)$ to predict the effect on the calibrant desorption kinetics from the extraction phase (Figure 7). Although the $k_{\mathrm{d}}$ values were close to one or more, which can be considered as labile, and have similar desorption kinetics, the lower $k_{\mathrm{d}}$ inhibits the release of calibrant from the extraction phase. Similar dependency of $k_{\mathrm{d}}$ was found in infinite volume cases (Figure S6b).

As discussed early, the extraction rate constant of the target analytes must vary to the same extent as the rate of the calibrant 
Table 1. Slopes Obtained from Variations in $a_{\mathrm{d}}$ with Respect to Changes in Free Analyte Concentrations in the Presence of BSA

\begin{tabular}{|c|c|c|c|c|c|c|}
\hline cmpd & $K_{\text {es }}$ & $K_{\mathrm{a}}(\mathrm{L} / \mathrm{kg})$ & $D_{\mathrm{s}} \times 10^{6}\left(\mathrm{~cm}^{2} / \mathrm{s}\right)$ & numerical simulation & exptl data ${ }^{24}$ & $\%$ error \\
\hline acenaphthene & 4211 & 4074 & 9.20 & 15.2 & $32.0 \pm 12.0$ & 52 \\
\hline phenanthrene & 8212 & 11220 & 8.80 & 6.07 & $7.49 \pm 2.12$ & 18 \\
\hline fluoranthene & 27020 & 42658 & 8.06 & 1.39 & $3.29 \pm 0.570$ & 57 \\
\hline pyrene & 29395 & 61660 & 7.33 & 1.27 & $1.32 \pm 0.142$ & 3 \\
\hline
\end{tabular}

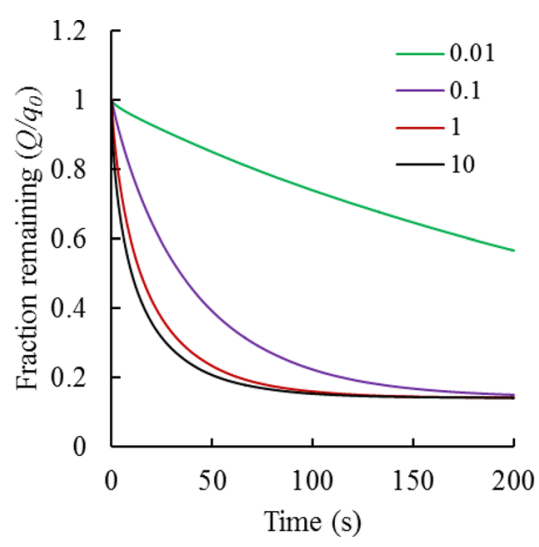

Figure 7. Effect of $k_{\mathrm{d}}\left(\mathrm{s}^{-1}\right)$ on the desorption kinetics. For all simulations, the $K_{\mathrm{a}}$ and $C_{\mathrm{M}}$ were kept constant at $1 \times 10^{5}$ and $0.1 \%$, respectively.

desorption in order to utilize CL-SPME for quantification of sample concentrations. Therefore, the model was used to investigate the change of $a_{\mathrm{e}}$ as a function of binding matrix concentration under all other constant experimental conditions. As expected from the theory of mass transfer, the extraction kinetics are mirrored with the corresponding desorption kinetics, as shown in Figure S8. The observed symmetry, regardless of matrix effects, confirms that any accelerated desorption kinetics of the calibrant are exactly compensated by a commensurate acceleration in extraction kinetics, thus validating the principle underlying the use of the preequilibrium CL-SPME approach.

Measurement of Total and Free Concentration. Once iso-symmetry is verified, calibration can be performed either by using the eq 4, where $K_{\text {es }}$ needs to be known or an external calibration curve. If the $K_{\mathrm{es}}$ is obtained from a matrix matched system, then the concentration is total; otherwise, the free concentration is obtained with the $K_{\mathrm{es}}$ measured from a binding-matrix free analyte solution. In order to verify that both the free and total concentrations can be obtained from the calibrant-loaded approach, an in-silico experiment using the developed computational model was carried out and the results are shown in the Supporting Information (Tables S1 and S2).

In cases where the $K_{\mathrm{es}}$ value is not available (for example, very hydrophobic chemicals that need very long equilibrium times), concentration of analytes can be obtained by using external calibration. Here, the sampling time used for constructing the calibration curve must be the same as that of the sample analysis. Although this approach is similar to the traditional external calibration method, the loaded standard serves as an internal standard to correct for variations in sample preparation, matrix effects, and detection processes. ${ }^{18}$ Also, this approach is suitable for very small volumes of sample, where addition of an internal standard in the sample is either troublesome or can change the sample characteristics. Consequently, if a matrix-matched external calibration curve is made with the use of a calibrant-loaded extraction phase, then the total concentration can be obtained, as shown in the Supporting Information (Figure S9). Since the extraction and desorption rates are enhanced by the matrix, free concentrations cannot be obtained with the use of a matrix-free standard calibration method that employs a calibrant-loaded extraction phase.

One-Calibrant Approach. In cases where stable isotopelabeled analogues of the target analytes are not available, Ouyang et al. ${ }^{25}$ demonstrated that it is possible to preload only one chemical that meets the criteria of a calibrant, and extrapolate the release kinetics on the basis of the physicochemical properties (e.g., $K_{\mathrm{es}}$ and $D_{\mathrm{s}}$ ) of the analyte/ calibrant couple, as shown by eq 7 :

$$
a_{\mathrm{e}}=a_{\mathrm{d}} \frac{D_{\mathrm{s}}^{\mathrm{A}} K_{\mathrm{es}}^{\mathrm{C}}}{D_{\mathrm{s}}^{\mathrm{C}} K_{\mathrm{es}}^{\mathrm{A}}}
$$

where the superscripts $\mathrm{A}$ and $\mathrm{C}$ refer to the analyte and calibrant, respectively, and $a_{\mathrm{e}}$ is the extrapolated extraction rate constant. Computational simulation results were compared with the experiments conducted by Ouyang et al. ${ }^{25}$ (Table 2). The use of only pyrene as a calibrant for the four chemicals provided theoretically precise quantification compared to the experimental values, whose deviation might be due to the associated experimental errors.

It should be noted that the analytes considered in the experiment (Table 2) have very close $D_{s}{ }^{A}$. The numerical model was also employed to study the effect of variation of $D_{\mathrm{s}}{ }^{\mathrm{A}}$ on the one calibrant approach. As shown in Table 3, unacceptably high error obtained from eq 7 can be reduced

Table 2. Validation of the Model with Experimental Data for the One-Calibrant Approach of SPME, Where Pyrene Was Considered as the Calibrant

\begin{tabular}{|c|c|c|c|c|c|c|}
\hline \multirow[b]{2}{*}{ analytes } & \multirow[b]{2}{*}{$K_{\mathrm{es}}$} & \multirow[b]{2}{*}{$D_{\mathrm{s}} \times 10^{6}\left(\mathrm{~cm}^{2} / \mathrm{s}\right)$} & \multicolumn{3}{|c|}{$a_{\mathrm{d}} \times 10^{-6} \mathrm{~s}^{-1}$} & \multirow[b]{2}{*}{$\%$ error $^{a}$} \\
\hline & & & exptl & numerical simulation & calcd from eq 7 & \\
\hline acenaphthene & 4266 & 7.66 & $44( \pm 1.1)$ & 37 & 38 & 3 \\
\hline anthracene & 9550 & 6.84 & $13( \pm 0.5)$ & 15 & 15 & 0 \\
\hline fluoranthene & 28626 & 6.59 & $4( \pm 0.2)$ & 4.9 & 4.8 & 2 \\
\hline pyrene & 40738 & 6.59 & $3.4( \pm 0.1)$ & 3.4 & 3.4 & 0 \\
\hline
\end{tabular}

$a_{\%}$ error $=\left|\frac{\text { data calculated from eq } 7-\text { experimental or numerical model data }}{\text { data calculated from eq } 7}\right| \times 100$ 
by considering the following modified semiempirical equation (eq 8):

$$
a_{\mathrm{e}}=a_{\mathrm{d}}\left(D_{\mathrm{s}}^{\mathrm{A}} / D_{\mathrm{s}}^{\mathrm{C}}\right)^{0.62} \times\left(K_{\mathrm{es}}^{\mathrm{C}} / K_{\mathrm{es}}^{\mathrm{A}}\right)
$$

Table 3. Effect of Diffusion Coefficient of Analytes $\left(D_{s}^{A}\right)$ on the Accuracy of the One Calibrant Equations (eq 7 and eq 8)

\begin{tabular}{cccccc} 
& \multicolumn{3}{c}{$a_{\mathrm{e}} \mathrm{min}^{-1} \times 10^{2}$} & & \\
\cline { 2 - 4 }$D_{\mathrm{s}}{ }^{\mathrm{A}} \times 10^{-6}$ & $\begin{array}{c}\text { numerical } \\
\text { simulation }\end{array}$ & $\begin{array}{c}\text { calcd } \\
\text { from } \\
\text { eq 7 }\end{array}$ & $\begin{array}{c}\text { calcd } \\
\text { from } \\
\text { eq 8 }\end{array}$ & $\begin{array}{c}\text { \% error } \\
\text { from eq 7 }\end{array}$ & $\begin{array}{c}\text { \% error } \\
\text { from eq 8 }\end{array}$ \\
1 & 1.82 & 1.82 & 1.82 & 0.00 & 0.00 \\
2.5 & 3.20 & 4.55 & 3.21 & 29.67 & 0.38 \\
5 & 4.93 & 9.10 & 4.94 & 45.82 & 0.13 \\
7.5 & 6.33 & 13.7 & 6.35 & 53.63 & 0.28 \\
10 & 7.55 & 18.2 & 7.59 & 58.52 & 0.49 \\
\hline
\end{tabular}

When employing the one-calibrant approach, one must answer the question of whether the calibrant has to be from the same class as the analytes under study. What if the $K_{\mathrm{es}}$ of the target analytes vary widely? In order to predict the suitability of the one-calibrant approach for a range of different target analytes, the mathematical model was utilized for varied target analytes so that a correlation could be assumed. In such cases, analytes with large molecular sizes or strong hydrophobicity may present a challenge due to the slow desorption of calibrants from the coating. Therefore, an upper limit (e.g., in $K_{\mathrm{ow}}$ ) needs to be established for the one-calibrant approach in CL-SPME applications. The mathematical model was further employed to study the limits of one-CL-SPME for the analysis of chemicals with a wide range of $K_{\mathrm{es}}$, as shown in Tables S3 and S4. The modified equation (eq 8 ) provided less than $4 \%$ error compared to eq 7 with an error of about 20\%. The observed results demonstrated that in cases where the calibrant was chosen from the middle of the range of $K_{\mathrm{es}}$ values of target analytes, the variation of $a_{\mathrm{d}}$ fell within the range of experimental error (assuming 20\% error). Therefore, the one-calibrant approach based on eq 8 can be said to be a suitable option for cases where the isotopically labeled calibrant is not available or not feasible to use.

The numerical model was also employed to study the performance of the one-calibrant approach for analytes with varied association constants with the binding matrix components present in the sample. The simulation results were used to formulate eq 9 for one calibrant approach with the presence of binding matrix component.

$$
a_{\mathrm{e}}=a_{\mathrm{d}}\left(D_{\mathrm{s}}^{\mathrm{A}} / D_{\mathrm{s}}^{\mathrm{C}}\right)^{0.62}\left(K_{\mathrm{es}}^{\mathrm{C}} / K_{\mathrm{es}}^{\mathrm{A}}\right)\left(K_{\mathrm{a}}^{\mathrm{A}} / K_{\mathrm{a}}^{\mathrm{C}}\right)^{0.85}
$$

As shown in Table S5, eq 9 provides accurate quantification even if the calibrant and analytes have different $K_{\mathrm{a}}$ values. It is worthwhile to mention that calibrant-free quantification is also possible at pre-equilibrium regimes of extraction with SPME as described by Ouyang et al. ${ }^{26}$

\section{CONCLUSIONS}

A comprehensive study of the calibrant-loaded extraction phase approach for quantitative chemical studies has been demonstrated with both experimental data and a computational model. The model simulation data not only aids in a better understanding of the inherent mechanisms and conditions of CL-EP approach of quantification but also predicts the essential parameters used for quantification. In this work, the iso- symmetric behaviors of sorption and desorption have been shown to be preserved for all variations of sample conditions, such as presence of a binding matrix, flow velocity, etc., in cases where both the calibrant and analyte interact identically with the coating. Nevertheless, for finite volume sample where the extracted amount is significant, a modified equation is proposed to obtain iso-symmetry. Further, the model can be used to predict desorption rate constants, which are needed for CL-EP quantification, of a wide range of target analytes with the use of only one calibrant for the correction of mass transfer properties, which is advantageous in cases where isotopically labeled calibrants are unavailable or their use not feasible. The results demonstrated that this CL-EP approach might solve the complexity due to the in vivo or in situ sample environment compared with the simplified in vitro release measurements carried out in buffer solutions. In particular, for a hydrophobic calibrant, where the calibrant release in the buffer is small or negligible, interactions with binding matrix components in real complex samples can alter the desorption profiles greatly. However, despite this complexity, the calibrant-loaded approach performs the necessary corrections while providing both free and total concentrations. In addition, the model can be used in predicting time weighted average (TWA) concentrations for SPME-based passive sampling. Moreover, use of the proposed model can aid in reducing both time and costs associated with experiments where long equilibration times are needed by predicting in silico rate constants.

\section{ASSOCIATED CONTENT}

\section{Supporting Information}

The Supporting Information is available free of charge on the ACS Publications website at DOI: 10.1021/acs.analchem.6b01802.

Schematics of sorption and desorption of calibrant and analytes, effect of diffusion coefficients on kinetics, and surface plots of the fluid flow around the SPME fiber; effect of matrix concentration; and tables for getting total and free concentrations (PDF)

\section{AUTHOR INFORMATION}

\section{Corresponding Author}

*Phone: +1-519-888-4641. Fax: +519 746 0435. E-mail: janusz@uwaterloo.ca.

\section{Notes}

The authors declare no competing financial interest.

\section{ACKNOWLEDGMENTS}

The authors thank the National Science and Engineering Research Council (NSERC) and Ontario Research Fund (ORF) of Canada for financial support. The authors also thank to Dr. Luis Ricardez-Sandoval of Department of Chemical Engineering, University of Waterloo, for his discussion in the initial development of the model.

\section{REFERENCES}

(1) Ouyang, G.; Pawliszyn, J. Anal. Chim. Acta 2008, 627, 184-97.

(2) Ter laak, T. L.; Busser, F. J. M.; Hermens, J. L. M. Anal. Chem. 2008, 80, 3859-3866.

(3) Chen, Y.; Pawliszyn, J. Anal. Chem. 2004, 76, 5807-5815.

(4) Zhou, N. S.; Zhang, X.; Ouyang, G.; Es-haghi, A.; Pawliszyn, J. Anal. Chem. 2007, 79, 1221-1230.

(5) Cui, X.; Bao, L.; Gan, J. Environ. Sci. Technol. 2013, 47, 9833-40. 
(6) Xu, B.; Chen, M.; Hou, J.; Chen, X.; Zhang, X.; Cui, S. J. Chromatogr. B: Anal. Technol. Biomed. Life Sci. 2015, 980, 28-33.

(7) Zhang, X.; Oakes, K. D.; Hoque, M. E.; Luong, D.; Metcalfe, C. D.; Pawliszyn, J.; Servos, M. R. Anal. Chem. 2011, 83, 3365-70.

(8) Booij, K.; Tucca, F. Mar. Pollut. Bull. 2015, 98, 365-7.

(9) Alam, M. N.; Ricardez-Sandoval, L.; Pawliszyn, J. Anal. Chem. 2015, 87, 9846-54.

(10) Siepmann, J.; Siepmann, F. J. Controlled Release 2012, 161, 35162

(11) Tcaciuc, A. P.; Apell, J. N.; Gschwend, P. M. Environ. Toxicol. Chem. 2015, 34, 2739-49.

(12) Zheng, X.; Bi, C.; Li, Z.; Podariu, M.; Hage, D. S. J. Pharm. Biomed. Anal. 2015, 113, 163-80.

(13) Ai, J. Anal. Chem. 1997, 69, 1230-1236.

(14) Kojic, M.; Milosevic, M.; Wu, S.; Blanco, E.; Ferrari, M.; Ziemys, A. Phys. Chem. Chem. Phys. 2015, 17, 20630-20635.

(15) Levich, V. Physicochemical Hydrodynamics. Prentice Hall: Englewood Cliffs, NJ, 1962.

(16) Thompson, J. M.; Hsieh, C. H.; Luthy, R. G. Environ. Sci. Technol. 2015, 49, 2270-7.

(17) Mayer, P.; Tolls, J.; Hermens, J. L.; Mackay, D. Environ. Sci. Technol. 2003, 37, 184A-191A.

(18) Wang, Y.; O’Reilly, J.; Chen, Y.; Pawliszyn, J. J. Chromatogr. A 2005, 1072, 13-17.

(19) Zhao, W.; Ouyang, G.; Alaee, M.; Pawliszyn, J. J. Chromatogr. A 2006, 1124, 112-20.

(20) Benhabib, K.; ter Laak, T. L.; van Leeuwen, H. P. Anal. Chim. Acta 2008, 609, 113-9.

(21) Benhabib, K.; Town, R. M; Leeuwen, H. P. Langmuir 2009, 25, $3381-3386$.

(22) Vrana, B.; Schuurmann, G. Environ. Sci. Technol. 2002, 36, 290296.

(23) Louch, D.; Motlagh, S.; Pawliszyn, J. Anal. Chem. 1992, 64, 1187-1199.

(24) Jiang, R.; Xu, J.; Zhu, F.; Luan, T.; Zeng, F.; Shen, Y.; Ouyang, G. J. Chromatogr. A 2015, 1411, 34-40.

(25) Ouyang, G.; Cui, S.; Qin, Z.; Pawliszyn, J. Anal. Chem. 2009, 81, $5629-5636$.

(26) Ouyang, G.; Cai, J.; Zhang, X.; Li, H.; Pawliszyn, J. J. Sep. Sci. 2008, 31, 1167-72. 